\title{
Design and experiment of automatic garlic seed directing device
}

\author{
Aijun Geng ${ }^{1,2^{*}}$, Xiaoyu $\mathrm{Li}^{1}$, Jialin Hou ${ }^{1}$, Ji Zhang ${ }^{1}$, Zhilong Zhang ${ }^{1}$, Jun Chong ${ }^{3}$ \\ (1. College of Mechanical and Electrical Engineering, Shandong Agricultural University, Taian 271018, Shandong, China; \\ 2. Shandong Key Laboratory of Horticultural Machinery and Equipment, Taian 271018, Shandong, China; \\ 3. Ji'nan Huaqing Agricultural Machinery Technology Co., Ltd., Ji'nan 251600, Shandong, China)
}

\begin{abstract}
Garlic planting requires a single seed with a scaly bud upward and vertically. In this study, an automatic garlic seed directing device was designed for auxiliary garlic seeding by utilizing a seed placed funnel, a correcting mechanism, and an automatic control system. Three factors influenced device performance: characteristics of garlic seeds, limited notch diameter of the seed placed funnel, and funnel taper of the seed placed funnel. The most optimal structural parameters of the seed placed funnel were determined via theoretical analysis. The research samples of Lanling and Jinxiang garlic were divided into three groups according to their respective sizes. 3D design software (Solidworks) was used to simulate the above three factors and analyze their rules of action. The obtained simulation results demonstrated that the suitable range of funnel taper and limited notch diameter of seed placed funnel for the three groups of seed were from $40^{\circ}$ to $60^{\circ}$ and $6-8 \mathrm{~mm}$, respectively. The bench test showed that when the funnel taper was $60^{\circ}$ and the limited notch diameter was $10 \mathrm{~mm}$, the success rates of the three groups' seeds in terms of scaly bud directing were $89.8 \%, 91.6 \%$ and $96.6 \%$ respectively, which satisfied the agricultural requirements of garlic planting. Accordingly, the corresponding results may promote the study and improvement of garlic intelligent planting equipment.
\end{abstract}

Keywords: garlic, garlic seed directing, simulation and analysis, automatic control DOI: $10.25165 /$ j.ijabe. 20201306.5142

Citation: Geng A J, Li X Y, Hou J L, Zhang J, Zhang Z L, Chong J. Design and experiment of automatic garlic seed directing device. Int J Agric \& Biol Eng, 2020; 13(6): 85-93.

\section{Introduction}

As one of the most important crops, garlic is widely cultivated in $\mathrm{China}^{[1]}$. In recent years, due to increasingly fierce international market competition, the garlic industry has made considerable strides and earned various victories. Therefore, the garlic industries' sustainable, healthy and stable development is of great significance. The agronomics of garlic planting requires planting seeds in scaly buds upward and vertically ${ }^{[2]}$. Due to such high requirements, farms have planted garlic through artificial means for a long time, which possesses many shortcomings like high labor intensity, low working efficiency and high economic costs. Above all, technical limitations have greatly restricted the development of the garlic industry. Certain planters have adopted mechanical seeding technology, where garlic seeds are directly transferred to an opened seed groove after taking the seeds ${ }^{[3]}$. Such planters include a garlic seeder with 3 to 12 rows from the United States ${ }^{[4,5]}$, hanging garlic planter produced by Bauchi, Spain, 3-row hanging garlic planter produced by ERME, France, 10-row garlic planter designed by Jiraporn et al. ${ }^{[6,7]}$ and 3-row garlic seeder

\section{Received date: 2019-05-12 Accepted date: 2020-05-15}

Biographies: Xiaoyu Li, MS, research interests: intelligent agricultural machinery equipment design, Email: 1xy940724@163.com; Jialin Hou, PhD, Professor, research interests: agricultural machinery design, Email: jlhou@sdau.edu.cn; Ji Zhang, PhD, Lecturer, research interests: design and theory of agricultural machinery, Email: sdauzhangii@163.com; Zhilong Zhang, $\mathrm{PhD}$, Lecturer, research interests: modern agricultural equipment and computer control, Email: zlzhang@163.com; Jun Chong, BS, Manager, research interests: garlic precision seeding equipment, Email: chongjun8866@163.com.

*Corresponding author: Aijun Geng, PhD, Associate Professor, research interests: design and theory of agricultural machinery. Shandong Agricultural University, Taian 271018, Shandong, China. Tel: +86-538-8242500, Email: gengaj@sdau.edu.cn. designed by Bakhtiari et al. ${ }^{[8]}$ However, this method cannot guarantee the orientation of the garlic seed scaly bud as its missed and replayed rate are greater than $5 \%$, inconsistent with the garlic sowing agronomy in China. Few garlic planters have a directional device, such as the YD-1500 garlic planter produced by the Korea Yongdong Company, which uses a disc duck-bill insertion device, where garlic seeds fall directly into the inserter through the seeding device while the upward rate of the bulbs was improved to a certain degree. The aforementioned test illustrated that the highest upward rate of the bulbs in the planter was $85 \%$ while its directing rate was low. Moreover, a plug-type garlic planting machine developed by Japan's Yanmar Company first manually placed seeds into seed trays, after which the seed trays were transported to the plot. This method still requires manual assistance and has high labor intensity. South Korea's Won-kyung Kim and colleagues designed a garlic vision-based precision seeder seed monitoring system as well as a seeding device based on machine vision. RGB cameras and optical sensors were integrated on the garlic seeder in order to capture the number of single and multiple seeds via imaging, the number of seeds and missing seeds may be used to separate multiple garlic seeds through a guided funnel, while simultaneously ensuring that garlic seeds are planted upright. The corresponding results demonstrated that the success rate of garlic seeds detection was $95 \%$. This system, however, has not been applied in actual production.

Most foreign garlic seeding machines are unable to adapt to the Chinese seeding environment, with the seeding effect being not ideal ${ }^{[9-13]}$. Domestic universities and scientific research institutions have carried out research on garlic seeding technology and achieved various results. Gao and Zhang ${ }^{[14]}$ designed a garlic seeding robot control system that accurately positions the seeding point during work. The designed algorithm may perform garlic seeding path planning, path calibration and real-time tracking 
functions. This study is currently experimental and has yet to be put into production. Cui et al. ${ }^{[15]}$ proposed judging the direction of large seeds using image processing technology. A CCD optical camera was used to collect images of the seeds, while images were sequentially processed for grayscale and binarization. By employing pumpkin seeds as the research subject, when the threshold value was 128 and the rectangle width was 10 pixels, the seed bulb direction was effectively identified. This study is currently in the laboratory test stage, providing new ideas for the research and design of image processing garlic orientation devices. Additionally, Zhao et al. ${ }^{[16]}$ proposed an orientation recognition algorithm for garlic cloves, where a camera captured the garlic clove's image, which was then processed using MATLAB so as to obtain a garlic clove contour. The scales and roots were judged by comparing the coordinate positions of the two points on the contour. Following experimentation, the recognition time of the algorithm was observed to be less than $0.5 \mathrm{~s}$, which is currently in the experimental research stage. A directing device with a conical coil spring seed tube was used in a garlic planter, where its rate of seed scaly bud upward was $91.3 \%{ }^{[17]}$. A shaker-type garlic seeder was designed by Tang et al. ${ }^{[18]}$ in which a single seed was taken using the garlic clove-shaped sieve holes on the spiral channel to clear the garlic seeds that do not meet the preset direction back to the seed box, allowing only garlic seeds in the same direction to pass through the channel. Since the shape and size of the garlic seeds were different, further optimizing the parameters of the shape of the sieve holes was necessary so as to improve the accuracy of garlic seed orientation. Xie et al. ${ }^{[19]}$ designed a garlic seeder planting mechanism in which garlic seeds were arranged in a row with scales facing the back of the bow. They were then pressed out of the garlic tube through the pressure plate and spring. However, the garlic seeds needed to be placed in the garlic pipe according to the sowing requirements in advance, resulting in large labor intensity. Furthermore, the shape and size of the garlic clove had a significant impact on the structural parameters of the mechanism, hence, additional improvements and optimizations were needed. Han et al. ${ }^{[20]}$ devised a garlic planter consisting of a garlic seed automatic orientation function. According to the overall dimensions of garlic seeds, a contoured pressing hole cone was designed. As the seeder advanced, seed holes with a shape similar to that of garlic seeds were pressed. The corresponding results showed that the planter's upward rate of buds was $96 \%$, however, the drop position of the garlic seeds as well as the formation of seed holes were inconsistent. Hou et al. ${ }^{[21,22]}$ developed a double duckbill garlic seeder that used a fixed duckbill receiving hopper to catch removed garlic seeds. The fixed hopper possessed an irregular opening at the bottom; if the garlic seeds were inverted, the scales may be extended. When the disc-type duckbill insertion bucket rotated, the receiving hopper was pushed longer on one side to open it. Normal garlic seeds can keep the scaly buds from falling into the planting bucket, and the inverted garlic bulbs were squeezed by the planting bucket. In this regard, the roots initially fell with the opening of the receiving hopper.

The aforementioned garlic seed directing devices utilized the interaction between garlic seeds and their mechanisms, which embodied its adequate performance for garlic seeds with obvious characteristics of scaly buds, such as Lanling garlic. However, for inconspicuous characteristics of scaly buds, such as Jinxiang garlic, these devices still have continued to have a low rate of scaly bud upward, which should be further improved.

Numerous investigations on garlic seed technologies were based on image processing and pneumatic types ${ }^{[23]}$. A garlic seed dividing and choosing device with image processing technology was designed with a rate of scaly bud upward of $92 \%$. A boundary following algorithm utilized USAN values and a crooked degree in order to recognize garlic seed scaly bud, in which the correct rate was determined to be $95 \%^{[24-27]}$. The above-mentioned technologies have been tested in the laboratory, though few reports exist regarding their transformation of achievements.

\section{Materials and methods}

\subsection{Structure}

The automatic garlic seed directing device proposed in the present study is composed of a seed placed funnel, correcting mechanism, automatic control system and frame. The structure of the device is shown in Figure 1. The overall parameters of the garlic seed directing device are shown in Table 1.

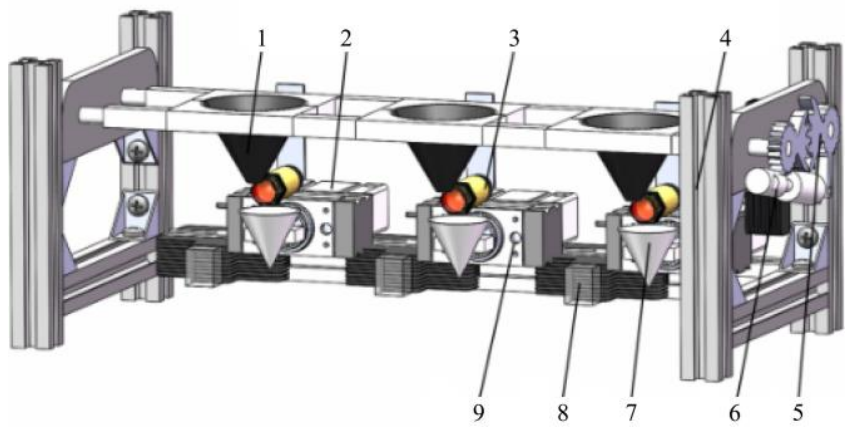

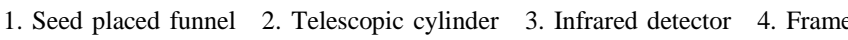
5. Driving gear 6. Open and close controlling cylinder 7. Rotational hopper 8. Solenoid directional control valve 9. Rotational cylinder

Figure 1 Automatic garlic seed directing device

Table 1 Main parameters of device

\begin{tabular}{lc}
\multicolumn{1}{c}{ Items } & Value/description \\
\hline Overall size/mm & $600 \times 350 \times 450$ \\
Material of funnel and hopper & Resin with 2 mm thickness \\
Material of frame & Aluminum alloy \\
Overall weight/kg & 15 \\
\hline
\end{tabular}

The seed placed funnel, driving gear and rotational hopper are self-restrained. The bottom of the seed placed funnel had a limited notch, only allowing garlic seed scaly to extend. The correcting mechanism included a rotational hopper, rotational cylinder and telescopic cylinder. The automatic control system included an infrared sensor, MCU, pneumatic solenoid valve, opening and closing control cylinder and air compressor. The part of the scaly bud that extended from the limited notch of the seed placed funnel could be detected by an infrared sensor, and the rotational hopper was driven horizontally by a telescopic cylinder and rotated $180^{\circ}$ using a rotational cylinder.

\subsection{Working principle}

The garlic seeds fell in a random manner and reached a stable state after falling into the seed placed funnel. If a scaly bud was upward, it would not be detected by the infrared detector, and the telescopic cylinder and rotational cylinder would be inoperable. Here, the MCU controls caused the seed placed funnel to open, where garlic seed fell into the inserted planting device directly. During this process, the infrared detector would not detect any signal. Conversely, if the scaly bud was downward, the scaly bud would have extended from the limited notch of the funnel after the seed fell into the seed placed funnel and be detected by the infrared 
sensor. Accordingly, the MCU would control the piston rod of the telescopic cylinder, enabling the rotational hopper to move below the seed placed funnel horizontally, which was driven by the solenoid directional control valve. In this regard, the seeds fell into the rotational hopper while the rotational cylinder rotated the garlic seeds $180^{\circ}$ so they would be adjusted when the scaly bud was upward.

\subsection{Characteristic analysis of garlic seeds}

The structure parameters of the seed placed funnel shares a significant relationship with the overall dimensions of garlic seeds. The completed and well-stacked Lanling and Jinxiang garlic seeds were chosen as the research subjects, as shown in Figure 2. The dimension distribution of the garlic seeds was counted and measured $^{[28,29]}$, as shown in Figure 3.

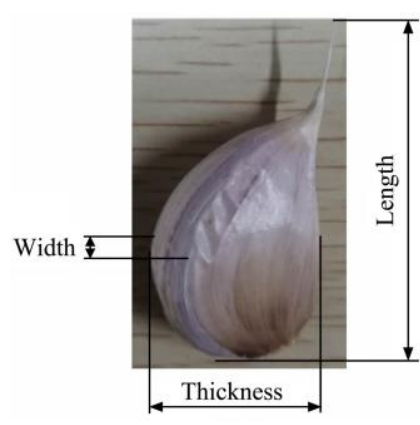

a. Lanling garlic



b. Type-I Jinxiang garlic

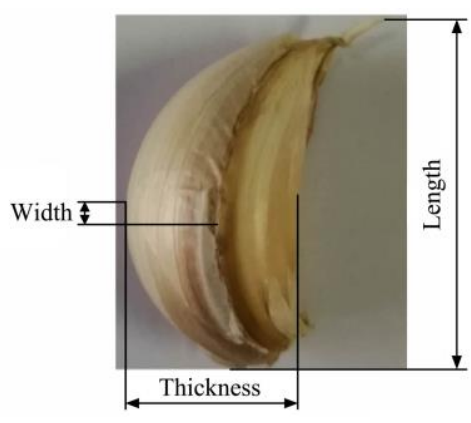

c. Type-II Jinxiang garlic

Figure 2 Two kinds of garlic seeds' overall dimensions

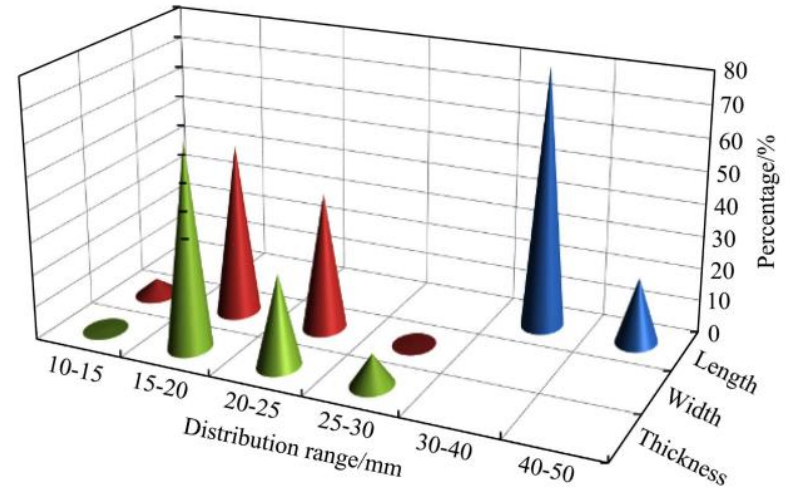

a. Lanling garlic

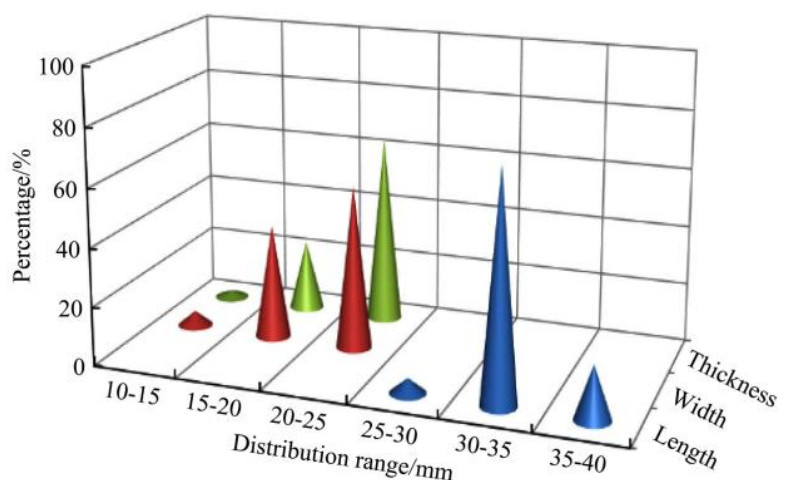

b. Type-I Jinxiang garlic



c. Type-II Jinxiang garlic

Figure 3 Distribution of the three groups of garlic seeds' overall dimensions

Jinxiang garlic seeds were divided into two groups according to shape and size, where the tip of the Type-I Jinxiang garlic seed was vertical to length direction, while the tip of the Type-II Jinxiang garlic seed was angled with the direction of length. However, the scaly bud of Lanling garlic seed grew along the direction of length.

According to the given results, the length of the Lanling garlic seed was mainly distributed between $30-40 \mathrm{~mm}$, accounting for $81 \%$, followed by $40-50 \mathrm{~mm}$ that accounted for $19 \%$. The width was distributed mainly within $15-25 \mathrm{~mm}$, where about $52.5 \%$ were distributed between $15-25 \mathrm{~mm}$ and $42 \%$ between $20-25 \mathrm{~mm}$. The thickness was distributed mainly within $15-25 \mathrm{~mm}$, among which $58 \%$ was distributed between $15-20 \mathrm{~mm}$ and $43.5 \%$ was distributed between $20-25 \mathrm{~mm}$. In regard to Type-I Jinxiang garlic, the length was mainly distributed within $30-35 \mathrm{~mm}$, accounting for $77 \%$. The width was distributed mainly between $20-25 \mathrm{~mm}$ and accounted for $56 \%$, while the thickness was distributed within $15-25 \mathrm{~mm}$, among which $24 \%$ was distributed within $15-20 \mathrm{~mm}$ and $64 \%$ was distributed between $20-25 \mathrm{~mm}$. As for Type-II Jinxiang garlic, the length was distributed within $30-35 \mathrm{~mm}$ at $54 \%$, while the width was mainly distributed between $10-20 \mathrm{~mm}$, among which $45 \%$ was distributed between $45 \%$ and $54 \%$ was distributed between $15-20 \mathrm{~mm}$. The thickness was distributed mainly within $15-20 \mathrm{~mm}$, accounting for $61 \%$.

The dimensions of the garlic seeds were measured using 150 seeds taken randomly from the samples by measuring their length $L$, width $W$ and thickness $D$ with Vernier Callipers, after which the average parameters were calculated using the following Equation 
(1):

$$
\bar{L}=\frac{\sum_{i=1}^{150} L_{i}}{150}, \bar{W}=\frac{\sum_{j=1}^{150} W_{j}}{150}, \bar{D}=\frac{\sum_{k=1}^{150} D_{i}}{150}
$$

where, $L$ is garlic seed length, $\mathrm{mm}$; $W$ is garlic seed width, $\mathrm{mm} ; D$ is garlic seed thickness, mm; $\bar{L}$ is average length of garlic seed, $\mathrm{mm} ; \quad \bar{W}$ is average width of rice seed, $\mathrm{mm} ; \bar{D}$ is average thickness of rice seed, mm.

The length distribution of Lanling garlic seeds were both uniform and concentrated, however, their width and thickness varied. The average of the length, width and thickness were $37.16 \mathrm{~mm}, 19.77 \mathrm{~mm}$ and $22.27 \mathrm{~mm}$, respectively. The difference in overall size of the Jinxiang garlic seed was obvious, while the average sizes of the Type-I Jinxiang garlic seed were $33.77 \mathrm{~mm}$, $19.99 \mathrm{~mm}$ and $21.68 \mathrm{~mm}$. Meanwhile, the average sizes of the Type-II Jinxiang garlic seeds were $32.45 \mathrm{~mm}, 13.61 \mathrm{~mm}$ and $17.74 \mathrm{~mm}$.

\subsection{Static analysis of garlic seeds in a seed placed funnel}

The main influential factors pertaining to length of scaly bud extension and current rate of detecting were found to be funnel taper $\theta$ and limited notch diameter $d$, as shown in Figure 4. Moreover, two main features were present for this funnel: (1) the funnel was designed to be an inverted cone shape with a taper. Garlic seeds can use the fraction between itself and the funnel as well as its gravity in order to adjust the direction of the scaly bud after falling at a freedom state from a fixed height. (2) The limited notch only allows the scaly bud of the seeds to extend when the scaly bud is faced downward, unlike the other seed. Here, orthographic projection of garlic seeds in a plate was used to establish a mathematical model of garlic seeds in a funnel. A support point was formed by the three ridge lines of the garlic seed on the max width of the funnel after the seed fell into the funnel.
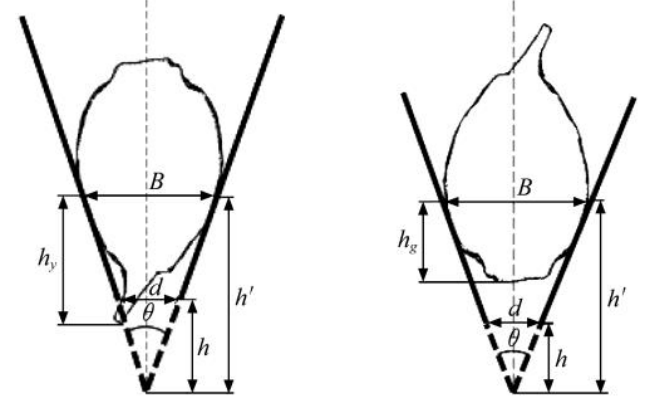

Figure 4 Two instances of the direction of scaly bud in the seed placed funnel

Letting the vertical distance between the limited notch and funnel tip to be $h$, the vertical distance between the support point and funnel tip is $h^{\prime}$, while the length of the scaly bud extending $h_{1}$ was represented by Equation (2). Also, the vertical distance $h_{2}$ between the root and limited notch was represented by Equation (3).

$$
\begin{gathered}
h_{1}=h_{y}-\left(h^{\prime}-h\right)=h_{y}-\frac{B-d}{2 \tan (\theta / 2)} \\
h_{2}=h^{\prime}-h-h_{g}=\frac{B-d}{2 \tan (\theta / 2)}-h_{g}
\end{gathered}
$$

where, $h_{y}$ represents the vertical distance between the scaly bud tip and support point, $\mathrm{mm} ; h_{g}$ represents the vertical distance between the seed root and support point, $\mathrm{mm} ; B$ represents the width of the garlic seed; $d$ represents the diameter of the limited notch, $\mathrm{mm}$; and $\theta$ represents the taper of the seed placed funnel, $\left({ }^{\circ}\right)$.

\section{Results and discussion}

\subsection{Simulation of garlic seed placed funnel}

\subsubsection{Simulation}

Considering the influence of the funnel taper as well as the limited notch diameter on the limit position of the garlic seed, seed placed funnels comprised of different parameters of combination were established using Solidworks, where the range of the limited notch was $5-15 \mathrm{~mm}$ with the step as $1 \mathrm{~mm}$, and the funnel taper was from $30^{\circ}-60^{\circ}$ with the step as $2^{\circ}$. Next, the $3 \mathrm{D}$ model of the garlic seed was imported in the funnel and adjusted to their relative positions so as to simulate the position between the scaly bud, root of garlic seed and limited notch of the funnel. The length of the scaly bud extending $h_{1}$ and vertical distance $h_{2}$ were measuring using the tools in Solidworks, as shown in Figure 5.
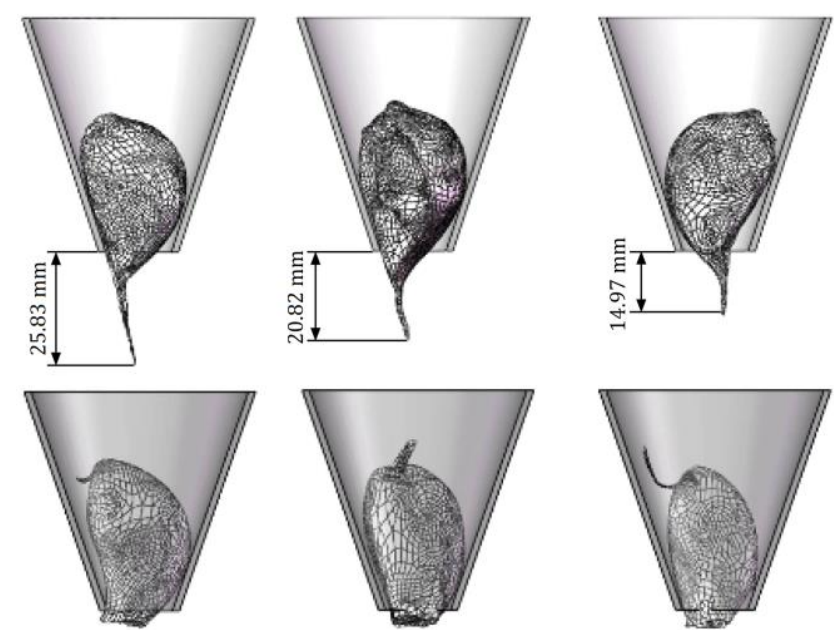

Figure 5 Simulation interface in Solidworks

\subsubsection{Analysis of simulation results}

The influence of the limited notch diameter and funnel taper of the seed placed funnel on the scaly bud and root of the garlic seed extending length were analyzed via MATLAB, Figure $6 a-6 c$ represent the exchange laws of the length of the scaly bud extending $h_{1}$ and vertical distance $h_{2}$ with the limited notch diameter and funnel taper of Type-I Jinxiang, Type-II Jinxiang and Lanling garlics, respectively.

According to the data analysis: (1) As the limited notch diameter was fixed, the vertical distance between the root and limited notch decreased gradually.

(2) When the hopper taper was fixed, and as the diameter of the limited notch increased, the limited position became closer to the limited notch. If the scaly bud faced downward, the length of the scaly bud extending became longer. If the scaly bud was faced upward, however, the distance between the root and limited notch became smaller, and the root was more likely to extend from the limited notch.

(3) Type-I of Jinxiang garlic possesses the characteristics of a short scaly bud and small root width. When $\theta \leq 35^{\circ}$, a small funnel taper limited the moving space of the inverted seed so that the scaly bud of most of the seeds cannot extend from the limited notch, while the extended part of a few of seeds were unable to be detected. When $\theta \geq 50^{\circ}$, the force of the wall of the funnel with the bigger taper for seeds disappeared, and the scaly bud declined or inverted, causing the length of the scaly bud extending to decrease. When $d \leq 5 \mathrm{~mm}$, the scaly bud was unable to extend from the limited notch, and when $d$ was close to $15 \mathrm{~mm}$, due to the diameter of the limited notch being bigger, the root of the seed with the scaly bud became upward and extended from the limited notch, resulting 
in incorrect detection by the sensor.

(4) With respect to Type-II Jinxiang garlic, when $d \geq 7.5 \mathrm{~mm}$, $\theta \geq 45^{\circ}$, the scaly bud extended from the limited notch, and when $\mathrm{d} \geq 10 \mathrm{~mm}, \theta \geq 50^{\circ}$, the root extended from the limited notch. (5) For Lanling garlic, when $\mathrm{d} \geq 5 \mathrm{~mm}, \theta \geq 40^{\circ}$, the scaly bud extended

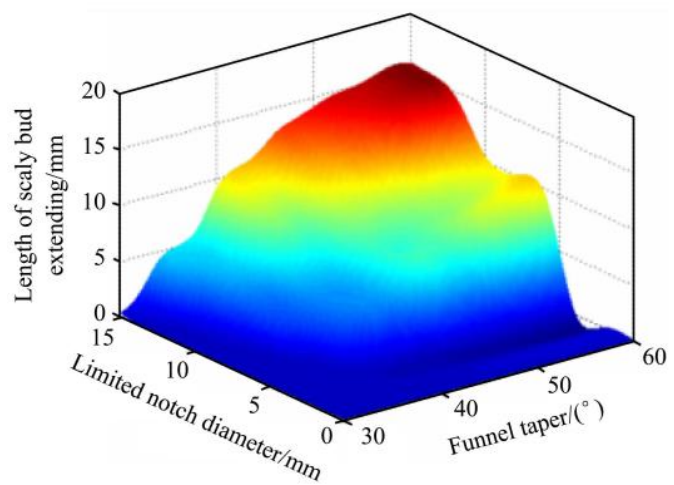

a. Analysis results of Type-I Jinxiang garlic

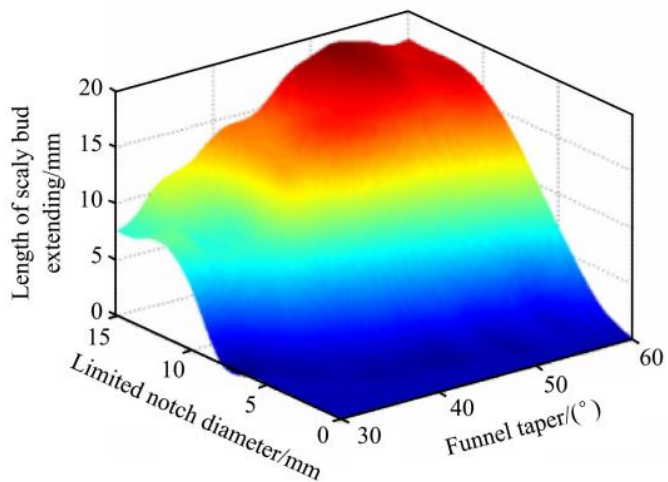

b. Analysis results of Type-II Jinxiang garlic

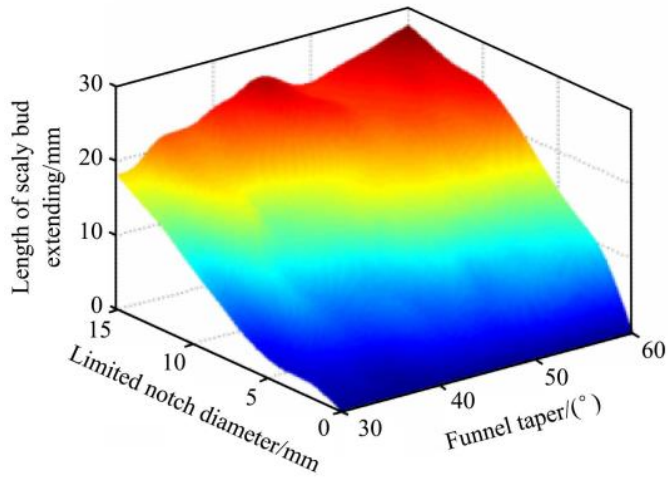

c. Analysis of Lanling garlic

Figure 6 Simulation results of the three groups of garlic

3.2 Determination of the seed placed funnel's structural parameters

\subsubsection{Test method}

The test of determination of the seed placed funnel's structural parameters was composed of a single factor and dual factor test. When the single test was carried out, 3D printing was used to print models of the funnels comprised of different structure parameters and develop single factor test of funnel taper and limited notch diameter. During testing, the three groups of garlic seeds fell into the funnel with the scaly bud being upward and downward, respectively. Afterward, the length of the scaly bud and root extending were counted. The purpose of this component was to further narrow down the range of the limited notch diameter and funnel taper, as shown in Figure 7.

The dual factors test was carried out by the seed placed funnel as well as a group of infrared sensors, as shown in Figure 8. First,
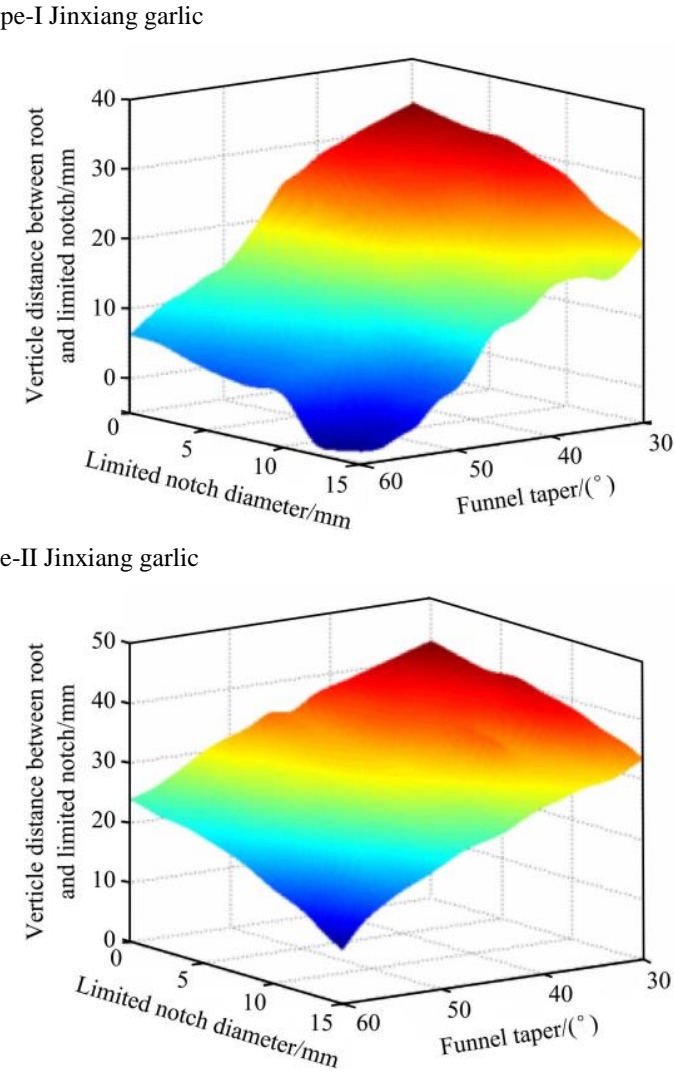

from the limited notch, which was dependent on the scaly bud being long. Additionally, when $\mathrm{d} \geq 10 \mathrm{~mm}$ and $\theta \geq 50^{\circ}$, the root extended from the limited notch. According to the above analysis, etermination of the structure parameters of the funnel should avoid too large a limited notch diameter and funnel taper.



the garlic seed fell into the funnel with the scaly bud upward. If the root was not detected, it fell into the funnel with the scaly bud downward for detection. Accordingly, the detected success rate of the scaly bud was taken by infrared sensor as an index. Here, if the infrared sensor generated a signal and created a record, this combination was excluded. Each test was repeated three times in order to eliminate any errors.

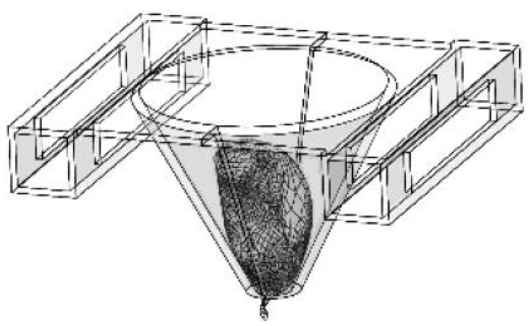

Figure 7 Test method of a single factor 



Figure 8 Detecting principle of the garlic seed scaly bud

\subsubsection{Single factor test results and analysis}

According to the simulation results, the funnel taper was divided into four levels: $30^{\circ}, 40^{\circ}, 50^{\circ}$, and $60^{\circ}$. Moreover, the bottom diameter was divided into four levels: $6 \mathrm{~mm}, 8 \mathrm{~mm}, 10 \mathrm{~mm}$, and $12 \mathrm{~mm}$. The trend of the length of the scaly bud and root extending with the funnel taper and limited notch diameter changed, as shown in Figure 9.

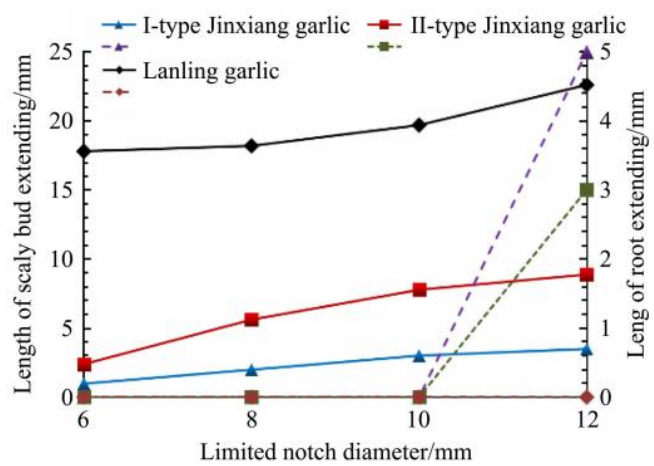

a. Influence of limited notch diameter on the scaly bud and root extending length

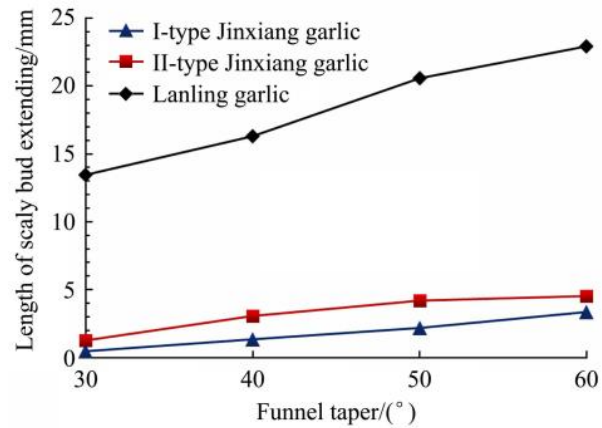

b. Influence of the funnel taper on the scaly bud extending length Note: The limited notch diameter was $10 \mathrm{~mm}$, and the funnel taper was $50^{\circ}$.

Figure 9 Results of the single factor test regarding the funnel structure's parameters

The results in Figure 9a demonstrate that when the funnel taper was $50^{\circ}$, the length of the garlic seed scaly bud gradually increased along with a rise in the limited notch diameter. As the scale bud of Jinxiang garlic was short and parallel to the level direction, the length of the scaly bud extending was much shorter than that of
Lanling garlic, which was consistent with the theoretical analysis. When the diameter of the limited notch was greater than $10 \mathrm{~mm}$, the roots of the three groups of garlic seeds protruded from the limited notch of the funnel, which did not meet the applicable requirements.

The results in Figure 9b show that when the limited notch diameter was $10 \mathrm{~mm}$, as the funnel taper increased, the length of the scaly bud extending gradually increased. When the funnel taper was less than $40^{\circ}$, the length of the scaly bud extending with regard to Jinxiang garlic was small, which was difficult to be detected by the infrared sensor. Hence, it did not meet the requirements for utilization, and the roots of the three groups of garlic seeds did not stick out of the limited notch of the seed placed funnel.

\subsubsection{Dual factors test and analysis}

A group of infrared sensors was installed in parallel to the limited notch when the seed placed funnel worked normally, as shown in Figure 10. Combined with the single factor test results, the structure parameters of the seed placed funnel was determined, where the funnel taper ranged between $40^{\circ}-60^{\circ}$ while the limited notch diameter ranged between $6-10 \mathrm{~mm}$. In order to further determine the best structure parameters of the seed placed funnel, dual factors as well as the three levels of funnel taper and limited notch diameter were carried out.

The test results are shown in Table 2, and the analysis of variance results are given in Table 3. According to the results regarding the range analysis in Table 2 , when the funnel taper was $60^{\circ}$ and the limiting notch diameter was $10 \mathrm{~mm}$, the detected success rate of the scaly bud of the Type-I Jinxiang garlic and II-type Jinxiang garlic was highest at $87.83 \%$ and $94.67 \%$, respectively. Additionally, Lanling garlic had a $100 \%$ detection success rate when the funnel taper was $50^{\circ}$ and $60^{\circ}$, while the limited notch diameter was $10 \mathrm{~mm}$.

According to the results on the variance analysis in Table 3, the funnel taper had a significant effect on Lanling garlic and II-type Jinxiang garlic with a judgment credibility of $90 \%$, which had no significant effect on Type-I Jinxiang garlic. Although the limited notch diameter had a significant effect on Type-I Jinxiang garlic with a judgment credibility as $95 \%$, it had no significant effect on Jinxiang garlic and II-type Jinxiang garlic. Evidently, the limited notch diameter plays a leading role in the detected success rate of garlic seed scaly buds. The larger the diameter, the larger the length of the scaly bud extending, and the higher the detected success rate. Considering the versatility of the funnel for the three types of garlic seeds, when combined with the single factor test of the funnel taper and limited notch diameter, the structure parameters of the seed placed funnel had a funnel taper of $60^{\circ}$ and a limited notch diameter of $10 \mathrm{~mm}$.

Table 2 Influence of notch diameter and funnel taper on directing success rate

\begin{tabular}{|c|c|c|c|c|c|}
\hline \multirow{2}{*}{ Number } & \multirow{2}{*}{ Funnel taper $/\left(^{\circ}\right)$} & \multirow{2}{*}{$\begin{array}{l}\text { Diameter of limited } \\
\text { notch/mm }\end{array}$} & \multicolumn{3}{|c|}{ Success rate of scaly bud was detected/\% } \\
\hline & & & Lanling garlic & Type-I Jinxiang garlic & Type-II Jinxiang garlic \\
\hline 1 & & 6 & 75.17 & 1.17 & 3.50 \\
\hline 2 & 40 & 8 & 86.14 & 11.00 & 61.33 \\
\hline 3 & & 10 & 95.33 & 19.83 & 75.50 \\
\hline 4 & & 6 & 94.83 & 5.33 & 73.67 \\
\hline 5 & 50 & 8 & 95.67 & 26.83 & 87.17 \\
\hline 6 & & 10 & 100 & 64.83 & 91.33 \\
\hline 7 & & 6 & 96.17 & 12.50 & 82.50 \\
\hline 8 & 60 & 8 & 98.33 & 73.00 & 92.00 \\
\hline 9 & & 10 & 100 & 87.83 & 94.67 \\
\hline
\end{tabular}


Table 3 Analysis of variance results

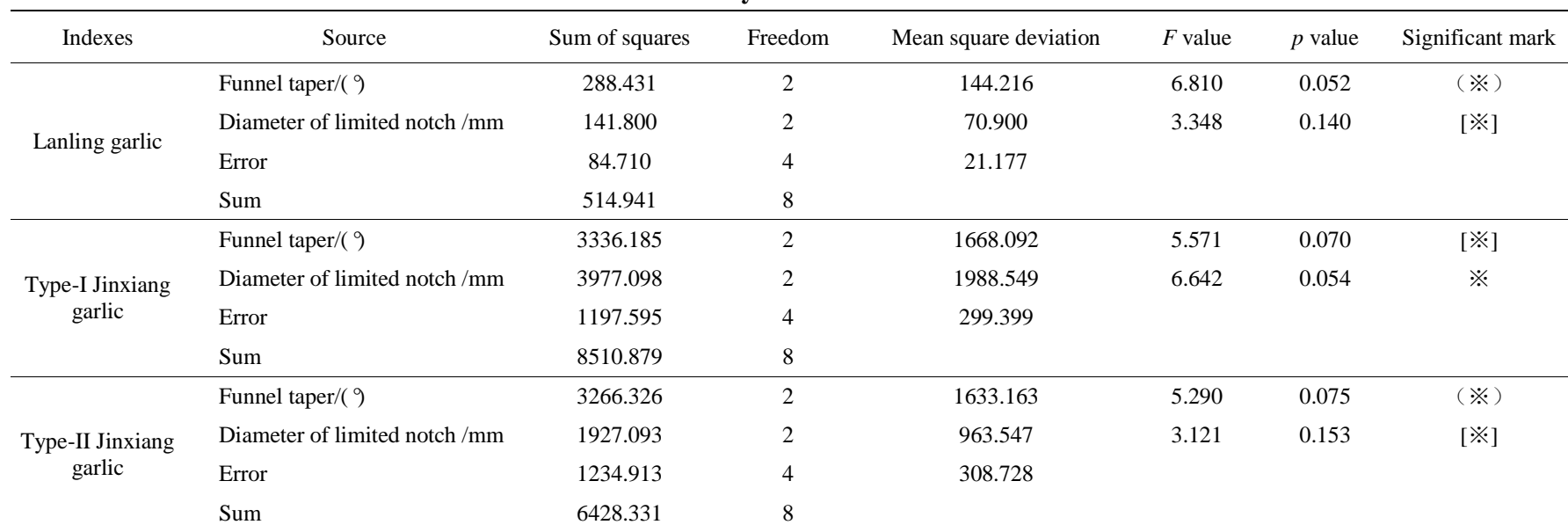

Note: $F_{0.25}(2,4)=2.00, F_{0.10}(2,4)=4.32, \quad F_{0.05}(2,4)=6.94, \quad F_{0.01}(2,4)=18.00 . \quad F_{0.01}(2,4) \geq F \geq F_{0.05}(2,4)$ represents the influence of factors on indexes being significant marked as "※”. $\quad F_{0.05}(2,4) \geq F \geq F_{0.1}(2,4)$ represents the influence of factors on indexes being more significant marked as "( $\left.※\right)$ ". $\quad F_{0.1}(2,4) \geq F \geq F_{0.25}(2,4)$ represents the influence of factors on indexes being not significant but influential and is marked as "[※]".

\subsection{Optimization test of the seed placed funnel}

The results illustrate that the detected success rate of the scaly bud of Jinxiang garlic was lower due to the direction of the scaly bud of Type-I Jinxiang garlic being mostly horizon with its length. Moreover, the direction of the scaly bud of Type-II Jinxiang garlic declined, and the direction of extending is random. If the direction of the scaly bud was relative to the direction of the infrared light emission of the sensor, it would be detected, otherwise, it would not be detected. Thus, an additional sensor group was added onto the original, as shown in Figure 10. Then, the test of success rate of the Jinxiang garlic was performed again, as shown in the results of Table 4.



Figure 10 Install position of infrared sensor

Table 4 Optimization result of detecting success rate of Jinxiang garlic seeds

\begin{tabular}{ccccc}
\hline Project & 1 & 2 & 3 & Average \\
\hline Type-I Jinxiang garlic & $96.5 \%$ & $97.5 \%$ & $98.0 \%$ & $97.33 \%$ \\
Type-II Jinxiang garlic & $98.3 \%$ & $100 \%$ & $100 \%$ & $99.43 \%$ \\
\hline
\end{tabular}

The results show that the success rate of Type-I Jinxiang garlic was $97.33 \%$, which improved by $9.50 \%$. The success rate of Type-II Jinxiang garlic was $99.43 \%$, which improved by $4.93 \%$. The optimized detection sensor had obvious improvements, however, the Type-I Jinxiang garlic's scaly bud was softer and finer, and the scaly bud was still unable to extend from the limited notch.

\subsection{Design of the automatic control system}

An automatic control system was used to detect scaly bud signals via infrared sensor and to adjust the scaly bud direction, as shown in Figure11a. The corresponding system included a MCU (STC 12C 5A60S2), infrared sensor with counter tube, rotational cylinder (SMC, MSQB10A), telescopic cylinder (TN16X30-S), pneumatic solenoid valve (ATM, 4V210), and air compressor
(Shanghai Jaguar Company, Z-0.036/8). The infrared sensor was sensitive to white but insensitive to black and can judge whether scaly bud is detected via different colors of seeds and funnels. The rotational cylinder controlled the rotation and reset the rotational hopper. The telescopic cylinder was a key component that enabled the rotational cylinder and rotational hopper to alternately move back and forth.

The workflow of the automatic control system is shown in Figure $11 \mathrm{~b}$, where the inputs of the first pneumatic solenoid valve, second pneumatic solenoid valve and third pneumatic solenoid valve is shown to be connected with an air compressor. Port P 0.0 of the MCU was the input of the sensor signal, while Port $\mathrm{P}$ 1.0-P1.2 were the outputs of the pneumatic solenoid values.

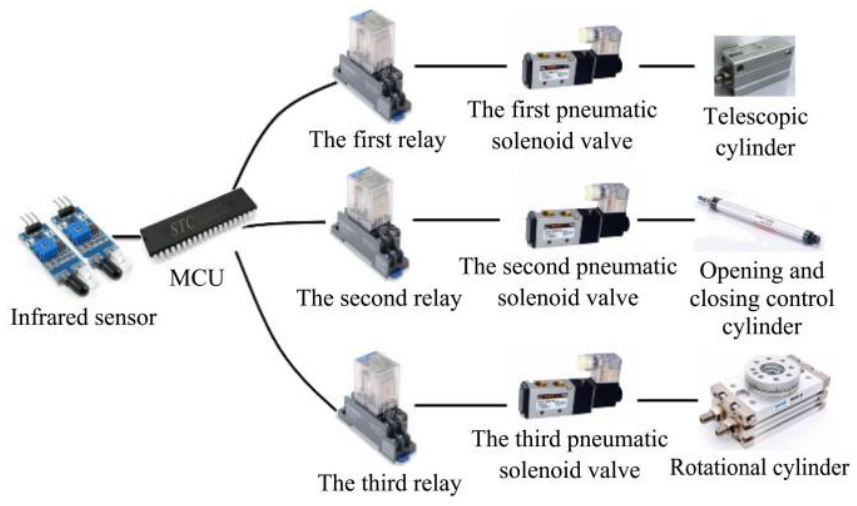

a. Structure of the control system

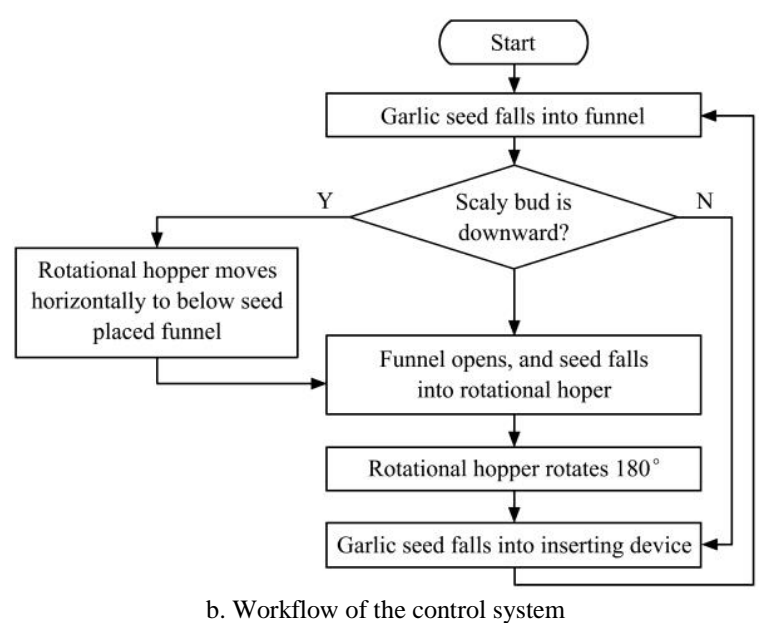

Figure 11 Structure and workflow of the control system 
The working principle of the proposed device is as follows. If the scaly bud of the inverted garlic seed extends from the limited notch, the line of the infrared sensor would be sheltered and reflected, triggering the MCU and exporting an electric signal to relays and pneumatic solenoid valves. Here, the inner circle of the first pneumatic solenoid value was electric, where air path changes and high air filled into the cylinder. The push rod then made the rotational hopper to horizontally move below the seed placed funnel. The second pneumatic solenoid value was electric, where the push rod of the opening and closing control cylinder enabled the seed placed funnel to open, while garlic seeds fell into the rotational hopper. Finally, the third pneumatic solenoid value was electric, where the rotational cylinder drove the rotational hopper to rotate $180^{\circ}$, while garlic seeds fell into the inserted device with a scaly bud faced upward.

\subsection{Experiment of the automatic garlic seed directing device}

In order to prove the entire working effect of the automatic garlic seed directing device, a directing test was carried out, as shown in Figure 12. During the test, an artificial method was chosen to send the seeds, where a transparent cone hopper was placed under a rotational hopper to easily count the rate of scaly buds being upward. The Lanling and Jinxiang garlics had 100 seeds selected, respectively, and the test was carried out five times. The results are given in Table 5 .
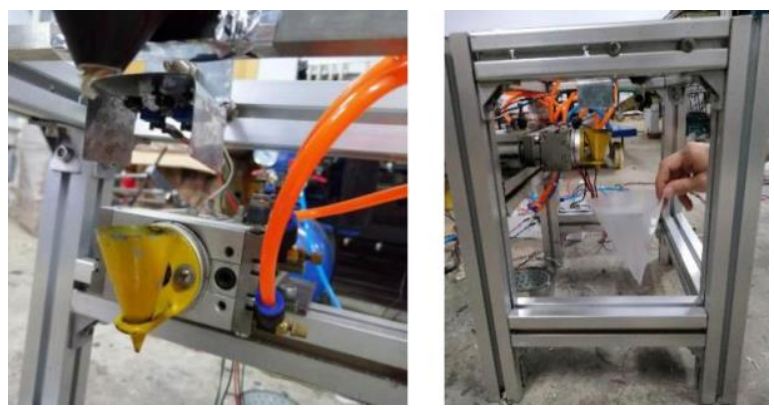

Figure 12 Test of garlic seed directing

Table 5 Results of the garlic seeds directing test

\begin{tabular}{cccc}
\hline & \multicolumn{3}{c}{ Rate of scaly bud is upward/\% } \\
\cline { 2 - 4 } No. & Type-I Jinxiang garlic & Type-II Jinxiang garlic & Lanling garlic \\
\hline 1 & 89 & 92 & 97 \\
2 & 92 & 90 & 95 \\
3 & 87 & 93 & 96 \\
4 & 91 & 92 & 99 \\
5 & 90 & 91 & 96 \\
Ave. & 89.8 & 91.6 & 96.6 \\
\hline
\end{tabular}

The test was stable, and the directing effect of the Lanling garlic was observed to be the best with the rate of scaly bud being upward of $96.6 \%$. The rate of scaly bud being upward of the Type-I Jinxiang garlic was $89.8 \%$, while that of Type-II Jinxiang garlic was $91.6 \%$. However, combining the hopper-type directing device and automatic directing device should be further developed.

\section{Conclusions}

This study proposed and devised an automatic garlic seed directing device that adjusted the direction of garlic seed scaly buds via presence or absence of detection signals. The structure and work parameters of each part were determined through a combination of theoretical analyses, simulations and experiments.

The proposed automatic garlic seed directing device included a seed placed funnel, rotational hopper, automatic control system and frame. This device automatically adjusted garlic seeds to bud upward. The seed placed funnel was designed according to the outline size of the seeds, where Lanling and Jinxiang garlic were divided into three groups as per their shape. The seed placed funnel had an inverted cone shape, limiting the vertical direction of the seed in the funnel. Moreover, the tip of the funnel had a limited notch that allowed the scaly bud of the inverted seed to extend, unlike the other parts of the seed.

3D models of the seeds of the Lanling and Jinxiang garlic were constructed on the computer according to the physical and mechanical parameters of the seeds. Next, simulations were done regarding the state of garlic seeds with free attitude in seed placed funnel. The length of the scaly bud extending $h_{1}$ and vertical distance between the root and limited notch $h_{2}$ were then measured. The range of the funnel taper was from $40^{\circ}$ to $60^{\circ}$, and the limited notch diameter was from 6 to $10 \mathrm{~mm}$, which satisfied the design requirements of the funnel.

The physical models of the seed placed funnel were made, and an automatic controlling system was designed along with an intelligent garlic seed orientation device. The optimal parameter combination of the funnel determined via experimentation was a funnel taper of $60^{\circ}$ and limited notch diameter of $10 \mathrm{~mm}$. The detected success rate of the Lanling garlic scaly bud was $100 \%$ while the rate of orientation success was $96.6 \%$. The detected success rate of the Type-I Jinxiang garlic seeds scaly bud was $97.33 \%$ while the rate of orientation success was $89.8 \%$. Additionally, the detected success rate of the Type-II Jinxiang garlic seed was $99.43 \%$ while the rate of orientation success was 91.6\%. Therefore, the designed automatic garlic seed directing device improved the effects on seed orientation.

\section{Acknowledgements}

Shandong Major Science and Technology Innovation Projects (2018CXGC0203); National Special Vegetable Industry Technology System Project (CARS-24-D-01); Shandong Agricultural University "Double-first-class" Collaborative Innovation Team of Horticultural Machinery Equipment (SYL2017XTTD07).

\section{[References]}

[1] Li X Y, Geng A J, Hou J L, Zhang M Y, Zhang J, Li W. Research status of garlic seeding machinery. Farm Machinery, 2017; 2: 105-107, 109.

[2] Liu J Z, Geng A J, Hou J L, Li X Y, Zhang J, Zhang Z L. Technology and research status on directional device of garlic. Journal of Chinese Agricultural Mechanization, 2018; 39(2): 22-26. (in Chinese)

[3] Труфляк Е В, Скоробогаченко И С, Сапрыкин В Ю. Hand drill of accurately-oriented planting for cloves of garlic and onions. Научный журнал КубГАУ, 2014; 104(10): 1-17. (in Russian)

[4] Nare B, Naik R K, Shrivastava A K, Prakash A. Design, development and evaluation of self propelled garlic (Allium Sativum L.) clove planter. Ama Agricultural Mechanization in Asia, Africa \& Latin America, 2010; 45(2):74-79.

[5] Nare B. Design development and evaluation of self-propelled garlic (Allium sativum L.) Clove planter. JNKVV, 2010.

[6] Jiraporn B, Hai S, Nobutaka I. Design and control of metering system and furrow openers for garlic planter. International Agricultural Engineering Journal, 2010; 19(2): 39-47.

[7] Jiraporn B, Hai S, Nobutaka I. Study of the mechanics of a $5 \mathrm{hp}$ power tiller attached to a 10-row garlic planter. Agricultural Mechanization in Asia, 2010; 41(1): 40-44.

[8] Bakhtiari M R, Loghavi M. Development and evaluation of an innovative garlic clove precision planter. Journal Agricultural Science Technology, 2009; 11(2): 125-136.

[9] Manjunatha M, Samuel D V K, Anurag R K, Gaikwad N. Development and performance evaluation of a garlic peeler. Journal of Food Science and Technology, 2014; 51(11): 3083-3093. 
[10] Gajakos A V, Saraf V V, Sneha S. Performance evaluation of manually operated garlic planter. International Journal of Agricultural Engineering, 2015; 8(1): 31-38.

[11] Bakhtiari M R, Amad D. Determining physical and aerodynamic properties of garlic to design and develop of a pneumatic garlic clove metering system. Agricultural Engineering International: CIGR Journal, 2015; 17(1): 59-67.

[12] Kumar D, Kumar J, Kant K. Studies and performance of a garlic planter in Uttar Pradesh. International Journal of Agricultural Engineering, 2017; 10(1): 228-223.

[13] Bahnasawy A H. Some physical and mechanical properties of garlic. International Journal of Food Engineering, 2007; 3(6): 1-20.

[14] Gao C, Zhang H. Study on the direction identification and alignment of garlic scaly bud. Advanced Materials Research. Trans Tech Publications Ltd, 2012; 482: 220-223.

[15] Cui Y J, Ding X, Li P P, Su S. Judgment of large seeds direction based on image processing. Journal of Agricultural Mechanization Research, 2012; 34(11): 125-128. (in Chinese)

[16] Zhao L Q, Ma Z Y. The study of garlic machine installation system of the directional recognition algorithm. Journal of Agricultural Mechanization Research, 2013; 35(6): 163-166. (in Chinese)

[17] Li R C, Sun X, Song C T, Zuo Z J, Du A F, Yue B P. Summary and research of model WZ-4 garlic transplanting machine. Agricultural Equipment \& Vehicle Engineering, 2013; 51(5): 15-17. (in Chinese)

[18] Tang T M, Zou S, Liu Y L, Chen J H, Xi G N. The application of vibration sieve and metering device in the garlic seeder upright screening device. Journal of Agricultural Mechanization Research, 2015; 37(12): 92-96. (in Chinese)

[19] Xie X H, Zhang Y, Liu Z, Chen Y J, Liu C X, Bi L G T. Design of planting mechanism for garlic planter. Transactions of the CSAE, 2015; 31(1): 34-39. (in Chinese)
[20] Han Q Y, Wang X Y, Hao J, Xie L J, Yu J. Design research of plug-hole automatic orientation garlic planting machine. Journal of Agricultural Mechanization Research, 2016; 38(7): 172-175. (in Chinese)

[21] Hou J L, Tian L, Li T H, Niu Z R, Li Y H. Design and experiment of test bench for garlic bulbil adjustment and seeding based on bilateral image identification. Transactions of the CSAE, 2020; 36(1): 50-58. (in Chinese)

[22] Hou J L, Huang S H, Niu Z R. Mechanism analysis and test of adjusting garlics upwards using two duckbill devices. Transactions of the CSAM, 2018; 49(11): 87-96. (in Chinese)

[23] Pan L, Deng S J, Liu R H. Research on the identification of the roots of garlic base on pattern recognition. Journal of Agricultural Mechanization Research, 2010; 32(5): 51-54. (in Chinese)

[24] Li Y H, Wu Y Q, Li T H, Niu Z R, Hou J L. Design and experiment of adjustment device based on machine vision for garlic clove direction. Computers and Electronics in Agriculture, 2020; 174: 105513.

[25] Guo Y F. Research of the recognition method of garlic direction in planting machinery. Xi'an: Northwest University of Agriculture and Forestry Science and Technology, 2011. (in Chinese)

[26] Cui Z C, Song J L, Xu T, Li N, Cai S R. Development of a garlic planting device. Journal of Agricultural Mechanization Research, 2017; 39(11): 131-135. (in Chinese)

[27] Xu T, Song J L, Cui Z C, Li N, Cai S R, Sun Q, et al. Study on related traits for mechanical planting of garlic seeds. Journal of Agricultural Mechanization Research, 2018; 40(5): 137-141. (in Chinese)

[28] Jin C Q, Yuan W S, Wu C Y, Zhang M. Experimental study on effects of the bulbil direction on garlic growth. Transactions of the CSAE, 2008; 24(4): 155-158. (in Chinese)

[29] Liu J. Effects of different garlic varieties and bulbil seeding directions on growth characteristic and quality. Tai'an: Shandong Agricultural University, 2018. (in Chinese) 